\title{
Edge-based electric field formulation in 3D CSEM simulations: a parallel approach
}

\author{
Octavio Castillo Reyes, Josep de la Puente, Vladimir Puzyrev, and José María Cela \\ Computer Applications in Science \& Engineering \\ Barcelona Supercomputing Center \\ Barcelona, Spain \\ octavio.castillo@bsc.es
}

\begin{abstract}
This paper presents a parallel computing scheme for the data computation that arise when applying one of the most popular electromagnetic methods in exploration geophysics, namely, controlled-source electromagnetic (CSEM). The computational approach is based on linear edge finite element method in 3D isotropic domains. The total electromagnetic field is decomposed into primary and secondary electromagnetic field. The primary field is calculated analytically using an horizontal layered-earth model and the secondary field is discretized by linear edge finite element method. We pre-calculated the primary field through of an embarrassingly-parallel framework in order to exploit the parallelism and the advantages of geometric flexibility. The numerical-computational formulation presented here is able to work with three different orientations for the dipole or excitation source. Our code is implemented on unstructured tetrahedral meshes because are able to represent complex geological structures and they allow local refinement in order to improve the solution's accuracy. The code's performance is studied through a test of scalability.
\end{abstract}

Keywords-High Performance Computing, exploration geophysics, edge-based finite element, Controlled-source Electromagnetic Method.

\section{INTRODUCTION}

$\mathbf{I}$ $\mathrm{N}$ the geological context around the oil wells, the electric conductivity is a parameter that plays an important role. The Marine Controlled-Source Electromagnetic Method (CSEM) has emerged as a useful exploration technique for mapping offshore hydrocarbon reservoirs and characterizing gas hydrates bearing shallow sediments [2], [8], [9], [10], [12], [18], [14]. The 3D EM modeling requires solving diffusive Maxwell's equations in a discretized form. The most popular numerical methods for EM forward modeling are Finite Difference (FD), Finite Element Method (FEM) and Integral Equation (IE).

Between them , the FEM is more suitable for modeling EM response in complex geometries. In 3D surveys, the domain can be discretized using hexahedral or tetrahedron elements. The EM field within each element of the domain can be approximated by either linear or higher order polynomial basis functions.

The node-based FEM was applied in the past to model EM data by solving the coupled equations for the vector and scalar potentials and also for solving Maxwell's equations for electric and magnetic fields [15], [24], [25]. However, for 978-1-4799-6908-1/15/\$31.00 (c) 2015 IEEE accurate computations, the divergence free condition for the EM fields in the source free regions needs to be addressed by an additional penalty term, commonly called Gauge condition, to alleviate possible spurious solutions [13], [15].

As a result, in FEM the use of Edge-based FEM (EFEM), also called Nèdèlec elements, has become very popular for solving EM fields problems. In fact, EFEM is often said to be a cure for many difficulties that are encountered (particularly eliminating spurious solutions) and they are claimed to yield accurate results [13], [16], [21]. The basis functions of Nèdèlec elements are vectorial functions defined along the element edges at the center of each edge. The tangential continuity of either electric or magnetic field is imposed automatically on the element's interfaces while the normal components are still can be discontinuous [13]. As a result, EFEM has the capability to model the frequency/time domain EM fields in the inhomogeneous complex bodies at any resistivities contrasts and at any survey types.

In this paper, we present the field formulation of Maxwell's equations for a 3D marine CSEM scenario and its parallel calculation. The software package is based on an abstract data structure and is focused in three main tasks: the edge conductivity computation, the primary electric field computation and the electric field interpolation. Common FEM functions such as assembly, ordering nodes and elements and solvers are based on FEniCS project. This approach allows the use different kinds of solvers with little effort.

We structured the paper as follows: in section two we describe the background theory of CSEM. In section three and four, we present the formulation of EM field equations in isotropic domains and the theory of Nèdèlec vectorial basis functions respectively. In section five we describe the main modules of our parallel framework to compute the EM field and its interpolation on unstructured tetrahedron meshes. Finally, an scalability test of the code is presented.

\section{MARINE CSEM}

Marine Controlled-source Electromagnetic Methods (CSEM) are a type of geophysical strategies to study the subsurface electrical conductivity distribution with an ample range of applications. CSEM techniques can be divided into two groups depending on the domain in which collected data is interpreted: time domains (TDEM) or frequency domains 
(FDEM). In the case of oil prospecting, marine CSEM surveys are done predominantly using FDEM [11], [14].

In marine CSEM, also referred as seabed logging [10], a deep-towed electric dipole transmitter is used to produce a low frequency EM signal (primary field) which interacts with the electrically conductive Earth and induces eddy currents that become sources of a new EM signal (secondary field). The two fields, the primary and the secondary one, add up to a resultant field, which is measured by remote receivers placed on the seabed. Since the secondary field at low frequencies, for which displacement currents are negligible, depends primarily on the electric conductivity distribution of the ground, it is possible to detect thin resistive layers beneath the seabed by studying the received signal [15]. Operating frequencies of transmitters in CSEM may range between 0.1 and $10 \mathrm{~Hz}$, and the choice depends on the dimensions of a model. In most studies, typical frequencies vary from 0.25 to $1 \mathrm{~Hz}$, which means that for source-receiver offsets of $10-12 \mathrm{~km}$, the penetration depth of the method can extend to several kilometres below the seabed [1], [2], [5], [15]. The disadvantage of marine CSEM is its relatively low resolution compared to seismic imaging. Therefore, marine CSEM is almost always used in conjunction with seismic surveying as the latter helps to constrain the resistivity model. Figure 1 depicts the marine CSEM. Marine CSEM is nowadays a well-known geophysical prospecting tool in the offshore environment and a commonplace in industry, examples of that can be found in [6], [9], [18], [14], [22].

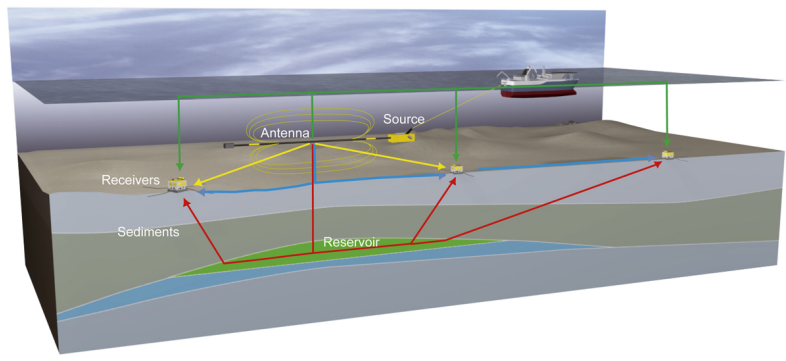

Fig. 1. Marine CSEM

Marine CSEM is a viable and cost-effective oil exploration technique. When integrated with other geophysics data, mainly seismic information, CSEM surveys are promising for adding value in shallow/deep waters. The outcomes and analysis of modeling with CSEM produce a more robust understanding of the prospection.

\section{EM FIELD FORMULATION}

In geophysical applications, the low frequency EM field satisfies the following Maxwell's equations:

$$
\begin{aligned}
& \nabla \times E=i_{\omega \mu_{0}} H \\
& \nabla \times H=J_{s}+\sigma E
\end{aligned}
$$

where we adopt the harmonic time dependence $e^{-i_{\omega t}}, \omega$ is the angular frequency, $\mu_{0}$ is the free space magnetic permeability, $J_{s}$ is the induced current in the conductive earth and $\sigma$ is the background conductivity. Actually, our formulation works for general isotropic domains.

In EM field formulations with FEM and EFEM, the anomalous formulations are desirable to overcome the mesh refinement in order to capture the rapid change of the primary current [4]. In the anomalous field formulation of EM field problem, the total field is decomposed into primary (background) and secondary fields [25]:

$$
\begin{aligned}
& E=E_{p}+E_{s} \\
& \sigma=\sigma_{p}+\Delta \sigma
\end{aligned}
$$

Based on this formulation, one can derive the following equation for the secondary electric field:

$$
\nabla \times \nabla \times E_{s}-i_{\omega \mu \sigma} E_{s}=i_{\omega \mu \Delta \sigma} E_{p}
$$

From 5, we can see that the source term for this equation is the primary electric field, which is much smoother than the source current. In this sense, our formulation is able to work with three different orientations for the primary excitation source or dipole, which are given by:

$\mathrm{X}$-directed dipole:

$$
\begin{aligned}
& E_{x}=\Upsilon \cdot\left[\frac{d_{x}^{2}}{r^{2}}\right] \cdot \Psi+k^{2} r^{2}-i k r-1 \\
& E_{y}=\Upsilon \cdot\left[\frac{d_{x} \cdot d_{y}}{r^{2}}\right] \cdot \Psi \\
& E_{z}=\Upsilon \cdot\left[\frac{d_{x} \cdot d_{z}}{r^{2}}\right] \cdot \Psi
\end{aligned}
$$

Y-directed dipole:

$$
\begin{aligned}
& E_{x}=\Upsilon \cdot\left[\frac{d_{x} \cdot d_{y}}{r^{2}}\right] \cdot \Psi \\
& E_{y}=\Upsilon \cdot\left[\frac{d_{y}^{2}}{r^{2}}\right] \cdot \Psi+k^{2} r^{2}-i k r-1 \\
& E_{z}=\Upsilon \cdot\left[\frac{d_{y} \cdot d_{z}}{r^{2}}\right] \cdot \Psi
\end{aligned}
$$

Z-directed dipole:

$$
\begin{aligned}
& E_{x}=\Upsilon \cdot\left[\frac{d_{x} \cdot d_{z}}{r^{2}}\right] \cdot \Psi \\
& E_{y}=\Upsilon \cdot\left[\frac{d_{z} \cdot d_{y}}{r^{2}}\right] \cdot \Psi \\
& E_{z}=\Upsilon \cdot\left[\frac{d_{z}^{2}}{r^{2}}\right] \cdot \Psi+k^{2} r^{2}-i k r-1
\end{aligned}
$$

with $\Upsilon$ and $\Psi$ determined by:

$$
\begin{aligned}
& \Upsilon=\frac{I \cdot d S}{4 \pi \sigma r^{3}} \cdot e^{-i k r} \\
& \Psi=-k^{2} \cdot r^{2}+3 i k r+3
\end{aligned}
$$


where $I$ is the dipole current, $d S$ is the dipole length, $\sigma$ represent the background conductivity, $k$ is the propagation parameter (wavenumber), $r$ is the module between source position and evaluation point position, $\left(d_{x}, d_{y}, d_{z}\right)$ is the distance between source position and evaluation point position. In EFEM formulations, the evaluation points are given by the mid-point for each edge in the mesh.

\section{EdGe-BASEd Finite Element Method}

The EFEM uses vector basis functions defined on the edges of the corresponding elements. Similar to the conventional node-based FEM, the domain can be discretized using rectangular, tetrahedron, hexahedron or other complex elements [3], [13].

Our solution is based on linear tetrahedron elements (six edges per element). Figure 2 is an illustration of the tetrahedron edge element that we used with node and edge indexing.

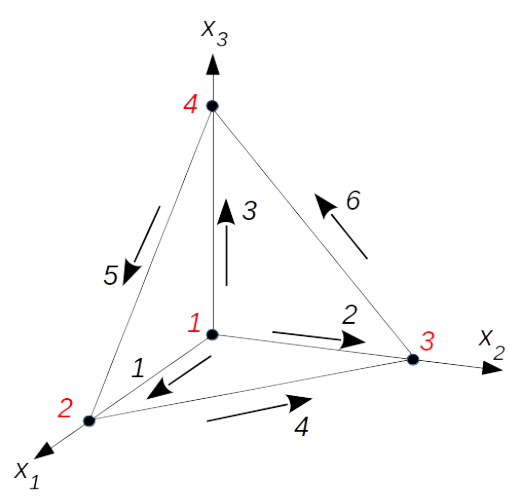

Fig. 2. An illustration of tetrahedron edge element. The red numbers indicates the node index and the black numbers are the index of edges.

Following the work by [13], the tangential component of the electric field is assigned to the mid-point of each edge of the corresponding element. Therefore, the electric field within each element can be approximated by:

$$
E^{e}=\sum_{i=1}^{6} E_{i}^{e} N_{i}^{e}
$$

where the coefficient $E_{i}^{e}$ corresponds to the projected tangential primary field over edge $i$ given by the dot product between $E_{p}$ and the unit vector of edge $i$, namely:

$$
E_{i}^{e}=E_{p_{x}} \cdot \hat{x}+E_{p_{y}} \cdot \hat{y}+E_{p_{z}} \cdot \hat{z}
$$

and $N_{i}^{e}$ are the conventional Lagrange interpolation functions [3], [13] for node-based FEM given by:

$$
N_{i}^{e}(x, y, z)=\frac{1}{6 V^{e}}\left(a_{i}^{e}+b_{i}^{e} x+c_{i}^{e} y+d_{i}^{e} z\right)
$$

TABLE I. EDGES DEFINITION FOR A TETRAHEDRAL ELEMENT

\begin{tabular}{ccc}
\hline Edge $i$ & Node $i_{1}$ & Node $i_{2}$ \\
\hline 1 & 1 & 2 \\
2 & 1 & 3 \\
3 & 1 & 4 \\
4 & 2 & 3 \\
5 & 4 & 2 \\
6 & 3 & 4 \\
\hline
\end{tabular}

If the edges numbers and their associated nodes $i 1$ and $i 2$ are defined as in table I, then equations 11 can be transformed into vector basis functions given by:

$$
N_{i}^{e}=W_{i 1 i 2} \ell_{i}^{e}=\left(\lambda_{i 1}^{e} \nabla \lambda_{i 2}^{e}-\lambda_{i 2}^{e} \nabla \lambda_{i 1}^{e}\right) \ell_{i}^{e}
$$

where $\ell_{i}^{e}$ is the length of edge $i$. The gradients for each basis function are given by the following expressions:

$$
\begin{aligned}
& \nabla \lambda_{1}^{e}=\frac{1}{6 V^{e}}\left|\begin{array}{l}
b_{1}^{e} \cdot \hat{i} \\
c_{1}^{e} \cdot \hat{j} \\
d_{1}^{e} \cdot \hat{k}
\end{array}\right| \quad \nabla \lambda_{2}^{e}=\frac{1}{6 V^{e}}\left|\begin{array}{l}
b_{2}^{e} \cdot \hat{i} \\
c_{2}^{e} \cdot \hat{j} \\
d_{2}^{e} \cdot \hat{k}
\end{array}\right| \\
& \nabla \lambda_{3}^{e}=\frac{1}{6 V^{e}}\left|\begin{array}{l}
b_{3}^{e} \cdot \hat{i} \\
c_{3}^{e} \cdot \hat{j} \\
d_{3}^{e} \cdot \hat{k}
\end{array}\right| \quad \nabla \lambda_{4}^{e}=\frac{1}{6 V^{e}}\left|\begin{array}{l}
b_{4}^{e} \cdot \hat{i} \\
c_{4}^{e} \cdot \hat{j} \\
d_{4}^{e} \cdot \hat{k}
\end{array}\right|
\end{aligned}
$$

Therefore, using barycentric coordinates defined by [3], [13], the expanded form of basis functions is the following:

$$
\begin{aligned}
& W_{12} \ell_{1}^{e}=\frac{1}{\left(6 V^{e}\right)^{2}}\left[\begin{array}{l}
b_{2}^{e}\left(\lambda_{1}\right)-b_{1}^{e}\left(\lambda_{2}\right) \cdot \hat{i} \\
c_{2}^{e}\left(\lambda_{1}\right)-c_{1}^{e}\left(\lambda_{2}\right) \cdot \hat{j} \\
d_{2}^{e}\left(\lambda_{1}\right)-d_{1}^{e}\left(\lambda_{2}\right) \cdot \hat{k}
\end{array}\right] \ell_{1} \\
& W_{13} \ell_{2}^{e}=\frac{1}{\left(6 V^{e}\right)^{2}}\left[\begin{array}{l}
b_{3}^{e}\left(\lambda_{1}\right)-b_{1}^{e}\left(\lambda_{3}\right) \cdot \hat{i} \\
c_{3}^{e}\left(\lambda_{1}\right)-c_{1}^{e}\left(\lambda_{3}\right) \cdot \hat{j} \\
d_{3}^{e}\left(\lambda_{1}\right)-d_{1}^{e}\left(\lambda_{3}\right) \cdot \hat{k}
\end{array}\right] \ell_{2} \\
& W_{14} \ell_{3}^{e}=\frac{1}{\left(6 V^{e}\right)^{2}}\left[\begin{array}{l}
b_{4}^{e}\left(\lambda_{1}\right)-b_{1}^{e}\left(\lambda_{4}\right) \cdot \hat{i} \\
c_{4}^{e}\left(\lambda_{1}\right)-c_{1}^{e}\left(\lambda_{4}\right) \cdot \hat{j} \\
d_{4}^{e}\left(\lambda_{1}\right)-d_{1}^{e}\left(\lambda_{4}\right) \cdot \hat{k}
\end{array}\right] \ell_{3} \\
& W_{23} \ell_{4}^{e}=\frac{1}{\left(6 V^{e}\right)^{2}}\left[\begin{array}{l}
b_{3}^{e}\left(\lambda_{2}\right)-b_{2}^{e}\left(\lambda_{3}\right) \cdot \hat{i} \\
c_{3}^{e}\left(\lambda_{2}\right)-c_{2}^{e}\left(\lambda_{3}\right) \cdot \hat{j} \\
d_{3}^{e}\left(\lambda_{2}\right)-d_{2}^{e}\left(\lambda_{3}\right) \cdot \hat{k}
\end{array}\right] \ell_{4} \\
& W_{42} \ell_{5}^{e}=\frac{1}{\left(6 V^{e}\right)^{2}}\left[\begin{array}{l}
b_{2}^{e}\left(\lambda_{4}\right)-b_{4}^{e}\left(\lambda_{2}\right) \cdot \hat{i} \\
c_{2}^{e}\left(\lambda_{4}\right)-c_{4}^{e}\left(\lambda_{2}\right) \cdot \hat{j} \\
d_{2}^{e}\left(\lambda_{4}\right)-d_{4}^{e}\left(\lambda_{2}\right) \cdot \hat{k}
\end{array}\right] \ell_{5} \\
& W_{34} \ell_{6}^{e}=\frac{1}{\left(6 V^{e}\right)^{2}}\left[\begin{array}{l}
b_{4}^{e}\left(\lambda_{3}\right)-b_{3}^{e}\left(\lambda_{4}\right) \cdot \hat{i} \\
c_{4}^{e}\left(\lambda_{3}\right)-c_{3}^{e}\left(\lambda_{4}\right) \cdot \hat{j} \\
d_{4}^{e}\left(\lambda_{3}\right)-d_{3}^{e}\left(\lambda_{4}\right) \cdot \hat{k}
\end{array}\right] \ell_{6}
\end{aligned}
$$

where $\lambda_{j}$ is given by:

$$
\lambda_{j}=a_{j}^{e}+b_{j}^{e} x+c_{j}^{e} y+d_{j}^{e} z \quad j=1 \ldots 4
$$


If $j$ is running in a cyclic way, coefficients $a_{j}^{e}, b_{j}^{e}, c_{j}^{e}, d_{j}^{e}$ can be obtained by:

$$
\begin{aligned}
a_{j}^{e} & =\left|\begin{array}{lll}
x_{j+1}^{e} & x_{j+2}^{e} & x_{j+3}^{e} \\
y_{j+1}^{e} & y_{j+2}^{e} & y_{j+3}^{e} \\
z_{j+1}^{e} & z_{j+2}^{e} & z_{j+3}^{e}
\end{array}\right| \\
b_{j}^{e} & =\left|\begin{array}{ccc}
1 & 1 & 1 \\
y_{j+1}^{e} & y_{j+2}^{e} & y_{j+3}^{e} \\
z_{j+1}^{e} & z_{j+2}^{e} & z_{j+3}^{e}
\end{array}\right| \\
c_{j}^{e} & =\left|\begin{array}{ccc}
1 & 1 & 1 \\
x_{j+1}^{e} & x_{j+2}^{e} & x_{j+3}^{e} \\
z_{j+1}^{e} & z_{j+2}^{e} & z_{j+3}^{e}
\end{array}\right| \\
d_{j}^{e} & =\left|\begin{array}{ccc}
1 & 1 & 1 \\
x_{j+1}^{e} & x_{j+2}^{e} & x_{j+3}^{e} \\
y_{j+1}^{e} & y_{j+2}^{e} & y_{j+3}^{e}
\end{array}\right|
\end{aligned}
$$

The computation of the coefficients $a_{j}^{e}, b_{j}^{e}, c_{j}^{e}, d_{j}^{e}$ must follow the checkerboard pattern defined by the expansion method [3], [13].

It is easy to verify that the vector edge basis functions are divergence free but not curl free, namely, $\nabla \cdot N_{i}^{e}=0$ and $\nabla \times$ $N_{i}^{e} \neq 0$. The vector edge basis functions are also continuous at the element boundaries. As a result, the divergence free condition of the electric field in the source free region and the continuity conditions are imposed directly in the EFEM formulation.

In order to obtain correct results and since the DOFs are integrals over edges, is necessary get an uniform global orientation. Namely, a positive orientation for each edge in the mesh is needed. To do that, our approach works as follows. If an edge adjoins two nodes $n_{i}$ and $n_{j}$, the direction of the edge as going from node $n_{i}$ to node $n_{j}$ if $i<j$. This simple algorithm gives a unique orientation of each edge in the mesh.

On the other hand, the local orientation of edges within an element is determined by his nodes indexes. For instance, the local edge direction for the element defined in table I are given by the vectorial function:

$$
S_{i}^{e}=\frac{\text { node }_{i 1}^{e}-\text { node }_{i 2}^{e}}{\mid \operatorname{node}_{i 1}^{e}-\text { node }_{i 2}^{e} \mid} \quad i=1 \ldots 6
$$

where $i$ is the edge index within element $e$ that adjoins $\operatorname{node}_{i 1}^{e}$ with $\operatorname{node}_{i 2}^{e}$.

\section{PARALLEL APPROACH}

Despite the popularity of the EFEM, there are few implementations of it. Furthermore, the 3D modeling of geophysical EM problems can easily overwhelm single core and modest multi-core computing resources [19]. To alleviate these issues, our parallel framework is based on an abstract data structure and is focused in three main tasks: the edge conductivity computation, the primary electric field computation and the electric field interpolation. Common FEM functions such as assembly, ordering nodes and elements and solvers are based in FEniCS [26]. This approach allows the use different kinds of solvers with little effort.
Actually, our software package is based on a shared memory parallel model defined by the OpenMP standard [20]. OpenMP has been widely adopted in the scientific computing community, and most vendors supports its Application Programming Interface (API) in their compiler suites. OpenMP offers not only parallel programs portability but, being based on directives, also a simple way to maintain a single code for the serial and parallel version of an application.

To exploit the advantages of geometric flexibility, our parallel approach is focused on embarrassingly parallel tasks related with the primary field computation. The main feature of this kind of parallelism is that does not exists data or communication dependency between these parallel tasks. In EFEM formulations the minimum level of computing work is related with the edges in the mesh. Therefore, our framework is integrated by the following modules:

1) Conductivity value $(\sigma)$ for each edge in the mesh

2) Primary field computation $\left(E_{p}\right)$

3) Electric field interpolation for each node in the mesh $E(x, y, z)$

The first two points are pre-processing tasks, while point 3 is a post-processing task. The algorithmic descriptions of each task are provided by algorithms 1,2 and 3 respectively. Parallel cycles are highlighted in yellow.

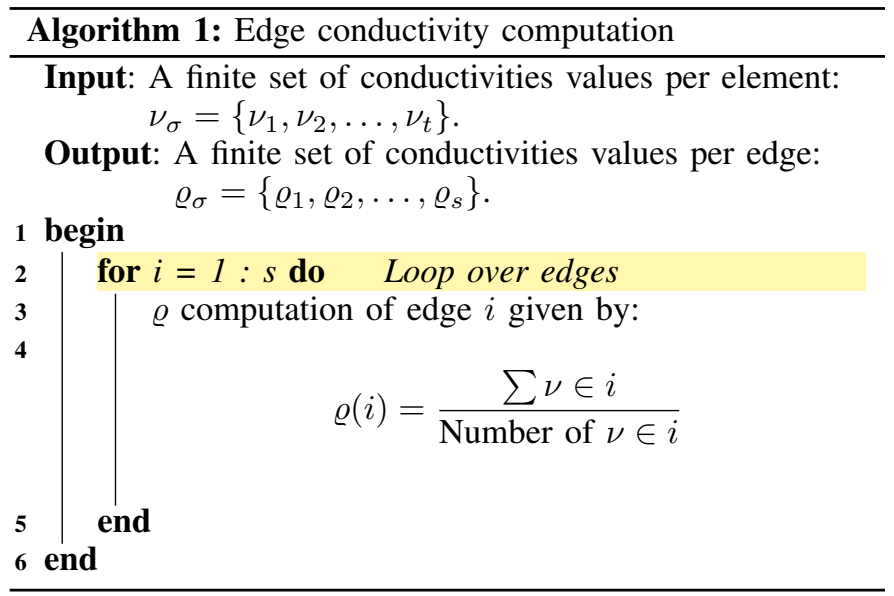

The embarrassingly parallelism in algorithms 1 and 2 is quite clear. On the other hand, algorithm 3 has two levels of parallelism, the first one is related with the nodes (outer loop) and the second one is related with the elements that sharing the node of outer loop. Since these loops are not squares, the use of OpenMP collapse-clause is not possible. Therefore, we parallelize the outer loops with a dynamic schedule in sake of a good load balancing.

\section{RESULTS AND SCALABILITY TEST}

To verify the accuracy and performance of our modeling, we used the in-line (the source and receivers are in the same line position) model of figure 3 which was defined by [7], [23]. The experiments were performed on the Marenostrum supercomputer with two 8 cores Intel Xeon processors E52670 


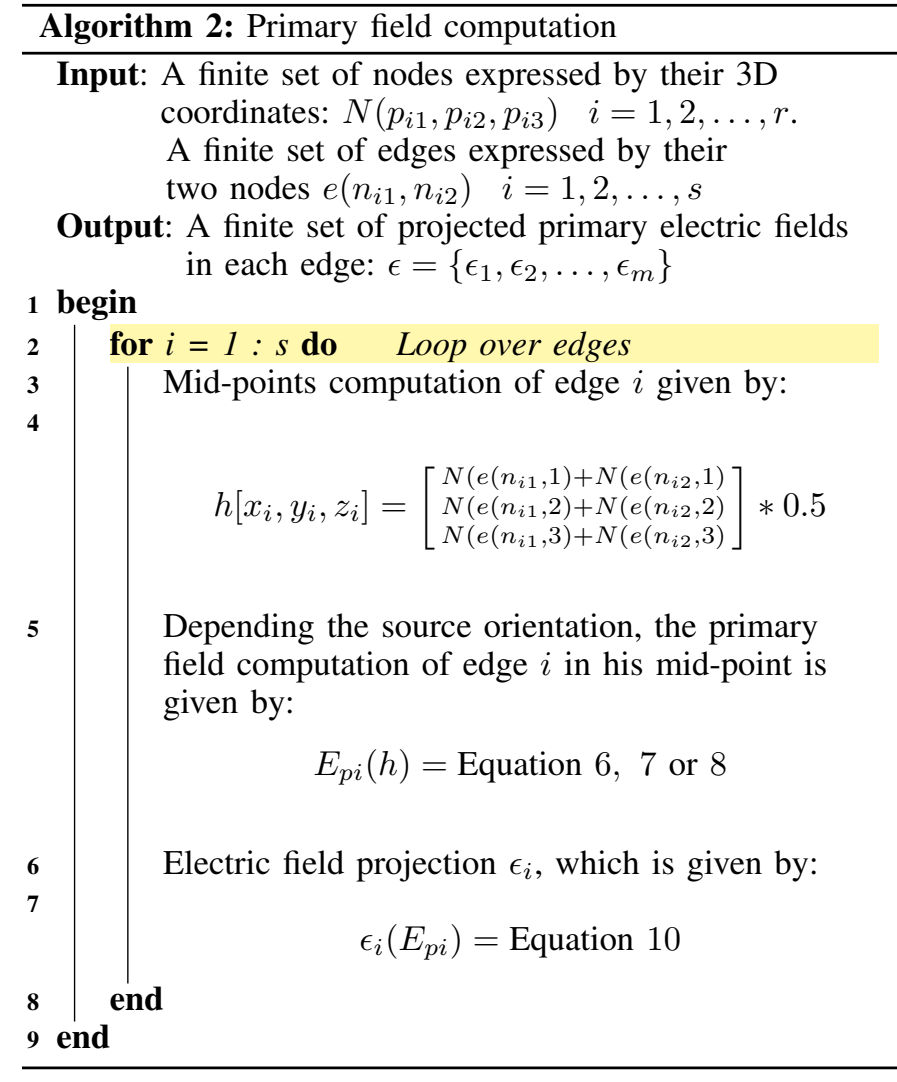

at 2.6 GHz per node. To increase the solution's accuracy, our implementation used an uniform refinement by Netgen mesh generator [17].

In order to validate our numerical formulation and the excitation source, we compute the primary field for a the background layered-earth model of figure 3 . The primary field of a x-directed dipole is show in figure 4. Sub-plots a, b and $\mathrm{c}$ in figure 4 show the electric field per component. Sub-plots $\mathrm{d}$, e and $\mathrm{f}$ figure 4 depict the rotation of the electric field in order to demonstrated the reciprocity theorem: the electric field produced by an horizontal electric dipole (HED) is equivalent to the azimuthal electric field generated by a vertical electric dipole (VED).

Table II presents a set of data obtained for various tests. For each test, the problem size stays fixed but the number of processing units are increased (strong scaling approach). The parallel scalability or parallel efficiency (as a percentage of linear) is given by:

$$
\chi=\frac{S}{n \cdot S n} \cdot 100
$$

where $S$ is the amount of time to complete a work unit with 1 processing unit, $n$ is the number of processing units and $S n$ is the amount of time to complete the same unit of work with $n$ processing units. In table II the time is expressed in seconds.

Figure 5 show the parallel scalability pattern of our framework. Tests 3 and 4 waste more cycles than tests 1 and 2

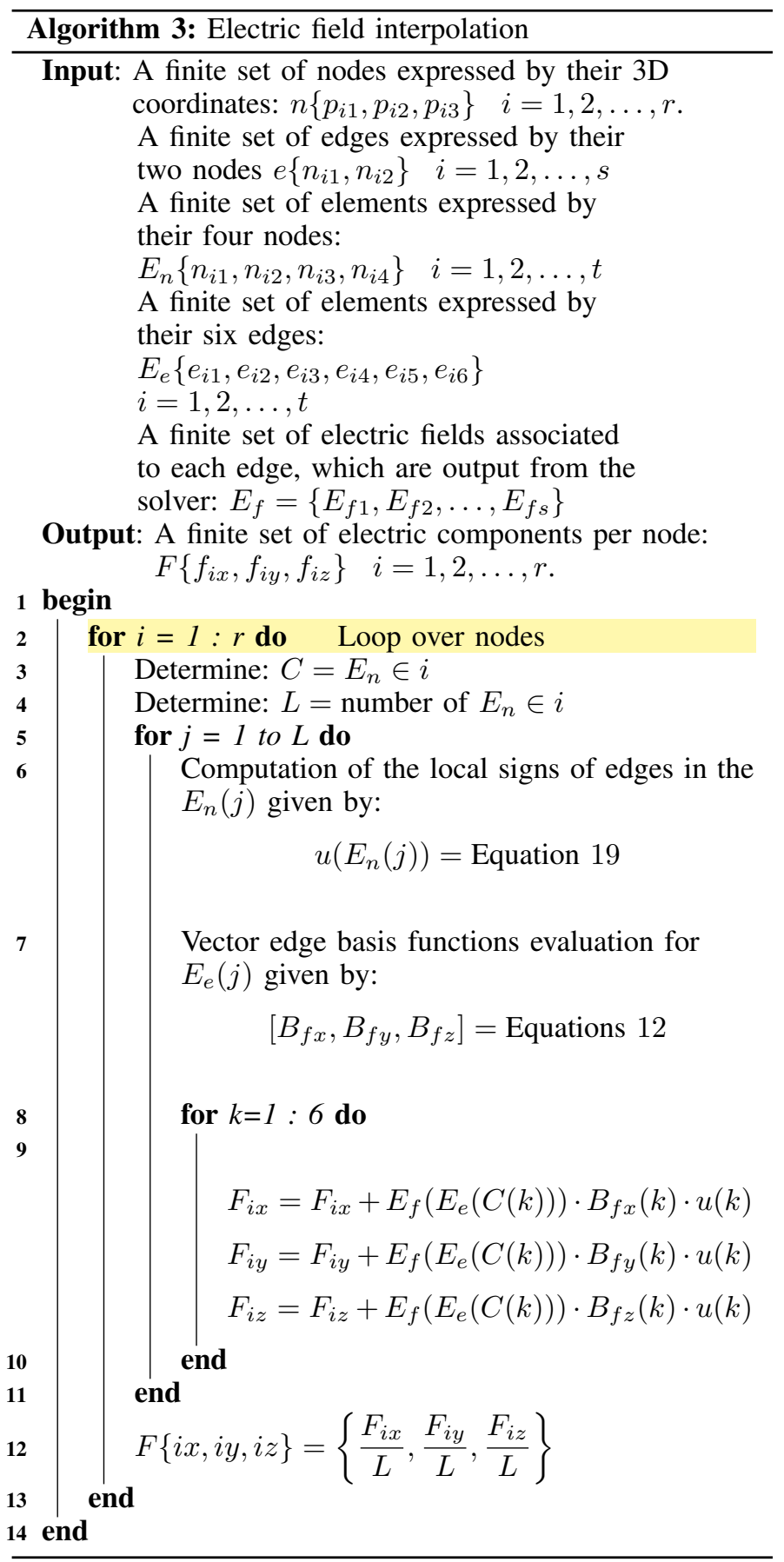




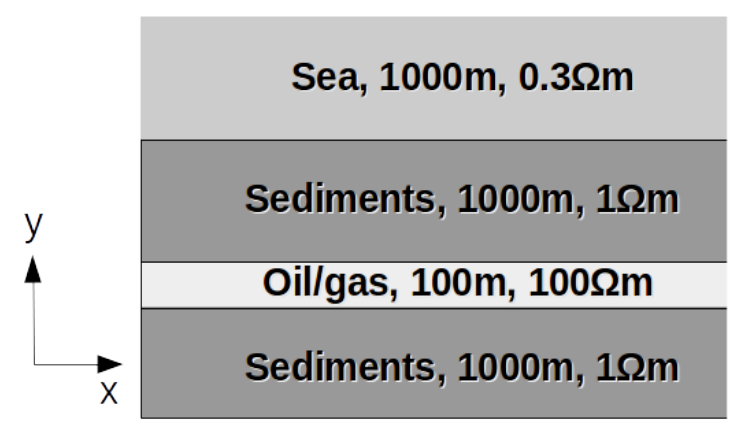

Fig. 3. Layer model (2D slice) (a)

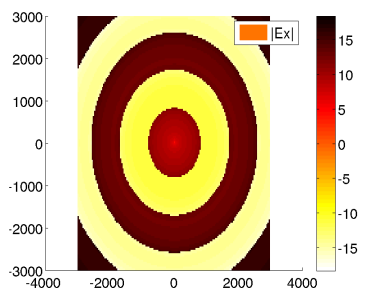

(b)

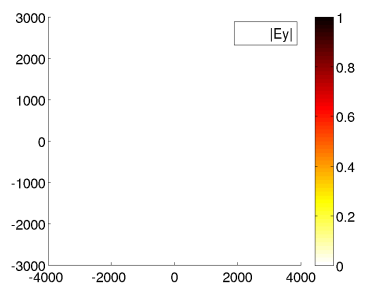

(c)

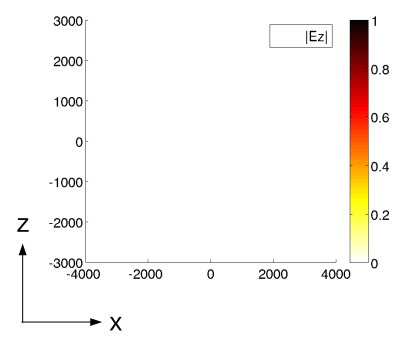

(d)

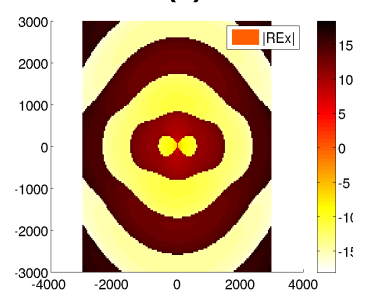

(e)

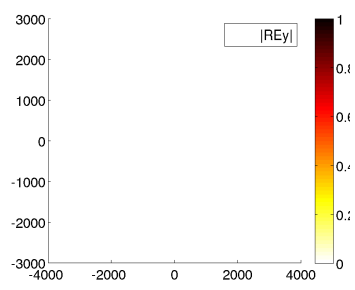

(f)

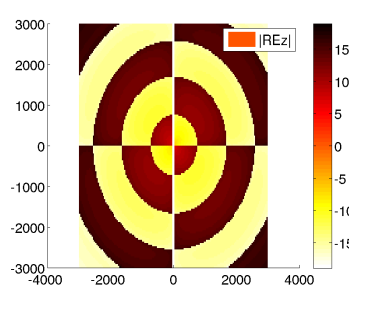

Fig. 4. Primary electric field for a x-dipole in an x-z plane. due to parallel overhead. A good lesson learned is that the communication overhead increases in proportion to the number of processes used. However, since our approach is based on embarrassingly parallel tasks, in our tests the communication overhead has no an important negative impact in the parallel scalability. As a result, we obtained a quasi linear speed-up, which is plotted in figure 6.

TABLE II. SUMMARY OF RESULTS

\begin{tabular}{|c|c|c|}
\hline \multicolumn{3}{|c|}{ Test 1: 3,072 elements, 4,184 edges } \\
\hline \# processors & Time & Parallel scalability (\%) \\
\hline 1 & 5.8705 & 100.00 \\
\hline 2 & 2.9500 & 99.50 \\
\hline 4 & 1.4938 & 98.25 \\
\hline 8 & 0.7526 & 97.50 \\
\hline 16 & 0.3837 & 95.62 \\
\hline 32 & 0.1977 & 93.12 \\
\hline 64 & 0.1010 & 91.41 \\
\hline \multicolumn{3}{|c|}{ Test 2: 24,576 elements, 31,024 edges } \\
\hline \# processors & Time & Parallel scalability \\
\hline 1 & 58.705 & 100.00 \\
\hline 2 & 29.799 & 98.50 \\
\hline 4 & 15.408 & 95.25 \\
\hline 8 & 7.9331 & 94.13 \\
\hline 16 & 4.0767 & 93.13 \\
\hline 32 & 2.0892 & 91.29 \\
\hline 64 & 1.0713 & 89.69 \\
\hline \multicolumn{3}{|c|}{ Test 3: 196,608 elements, 238,688 edges } \\
\hline \# processors & Time & Parallel scalability \\
\hline 1 & 763.17 & 100.00 \\
\hline 2 & 391.36 & 97.50 \\
\hline 4 & 205.70 & 94.75 \\
\hline 8 & 109.03 & 93.87 \\
\hline 16 & 56.531 & 92.63 \\
\hline 32 & 29.241 & 90.93 \\
\hline 64 & 15.196 & 89.41 \\
\hline \multicolumn{3}{|c|}{ Test 4: $1,572,864$ elements, $1,872,064$ edges } \\
\hline \# processors & Time & Parallel scalability \\
\hline 1 & $13,737.2$ & 100 \\
\hline 2 & $7,080.9$ & 97.2 \\
\hline 4 & $3,743.4$ & 94.3 \\
\hline 8 & $1,977.7$ & 90.2 \\
\hline 16 & $1,033.9$ & 89.1 \\
\hline 32 & $5,316.7$ & 87.4 \\
\hline 64 & $2,764.8$ & 85.2 \\
\hline
\end{tabular}

\section{CONCLUSIONS AND FUtURE WORK}

Marine CSEM, edge-based numerical formulation and its implementation on unstructured tetrahedral meshes were dis- 


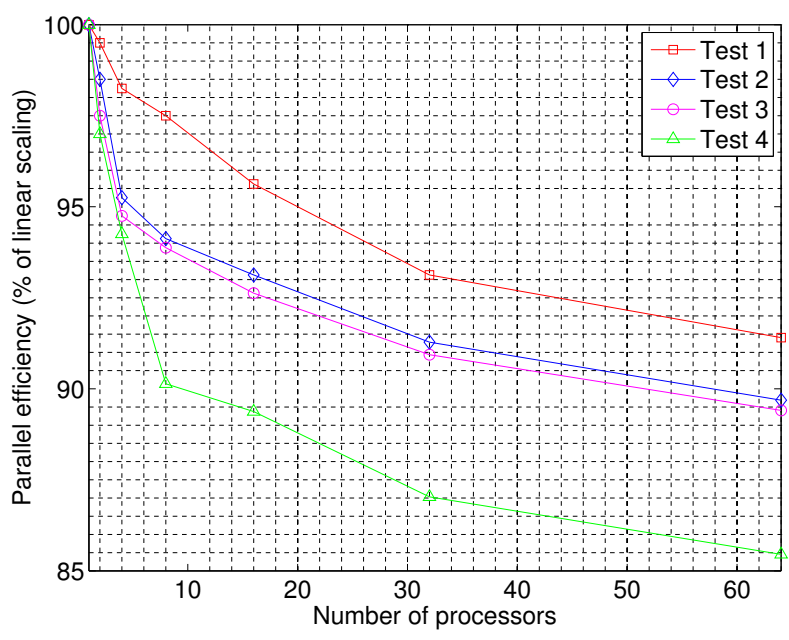

Fig. 5. Strong scaling test

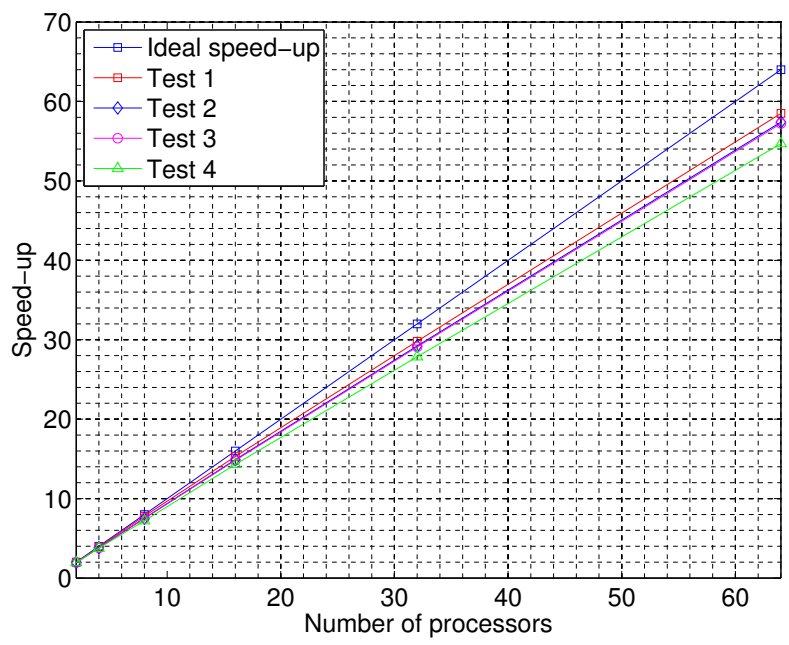

Fig. 6. Speed-up test

cussed. Marine CSEM is nowadays a well-known geophysical prospecting tool in the off-shore environment and a commonplace in industry. Then, we developed a parallel edge-based finite element algorithm to solve diffusive EM fields in 3D isotropic domains.

The use of vectorial basis functions (edge basis) instead of the node basis function (Lagrange basis) results in the FEM break-through as a tool to model the EM response accurately. Our numerical approach automatically enforce the divergence free conditions for EM fields. Moreover, the continuity of the tangential EM is satisfied automatically as well. Furthermore, the numerical formulation for the excitation source presented here is able to produce electric fields in any direction.

Our parallel framework is based on an abstract data structure with a strong emphasis in three main tasks of CSEM surveys: the edge conductivity computation, primary electric field computation and the electric field interpolation. Common FEM functions such as assembly, ordering nodes and elements and solvers are based on third party platforms. This approach allows the use different kinds of solvers with little effort.

The developed code was tested for different meshes (based on uniform refinement) of a typical layered-earth model of the hydrocarbons reservoir. The strong scaling test show that our parallel framework is profitable because the execution time is reduced. We achieved a quasi linear parallel efficiency. The test considers only the computation time of embarrassingly parallel tasks. In our experiments the minimum execution time is not limited by the communication overhead. As a result, we achieved a quasi linear speed-up.

Future work will be aimed in two lines. Firstly, at the implementation of an hybrid parallel model (MPI-OpenMP) to improve the computation time of the whole solution. Secondly, on adding more physical parameters such as topography, bathymetry and anisotropy.

\section{ACKNOWLEDGEMENTS}

This project has received funding from the European Union's Horizon 2020 research and innovation programme under the Marie Sklodowska-Curie grant agreement No. 644602.

Authors gratefully acknowledge the support from the Mexican National Council for Science and Technology (CONACYT).

\section{REFERENCES}

[1] Abubakar, A., Habashy, T., Druskin, V., Alumbaugh, D., Zerelli, A., Knizhnerman, L. (2006). Two-and-half-dimensional forward and inverse modeling for marine CSEM problems. In 2006 SEG Annual Meeting. Society of Exploration Geophysicists.

[2] Boulaenko, M., Hesthammer, J., Vereshagin, A., Gelting, P., Davies, R., and Wedberg, T. (2007). Marine csem technologythe luva case. Houston Geological Society.

[3] Burnett, D. S. (1987). Finite element analysis: from concepts to applications. Prentice Hall.

[4] Cai, H., Xiong, B., Han, M., Zhdanov, M. (2014). 3D controlled-source electromagnetic modeling in anisotropic medium using edge-based finite element method. Computers \& Geosciences, 73, 164-176.

[5] Carazzone, J. J., Burtz, O. M., Green, K. E., Pavlov, D. A., Xia, C. (2005). Three dimensional imaging of marine CSEM data. In 2005 SEG Annual Meeting. Society of Exploration Geophysicists.

[6] Commer, M., Newman, G. A. (2008). New advances in threedimensional controlled-source electromagnetic inversion.

[7] Constable, S., Weiss, C. J. (2006). Mapping thin resistors and hydrocarbons with marine EM methods: Insights from 1D modeling. Geophysics, 71(2), G43-G51.

[8] Constable, S. (2006). Marine electromagnetic methods: a new tool for offshore exploration. The Leading Edge, 25(4):438444.

[9] Constable, S. (2010). Ten years of marine csem for hydrocarbon exploration. Geophysics, 75(5):75A67-75A81.

[10] Eidesmo, T., Ellingsrud, S., MacGregor, L., Constable, S., Sinha, M., Johansen, S., Kong, F., and Westerdahl, H. (2002). Sea bed logging (SBL), a new method for remote and direct identification of hydrocarbon filled layers in deepwater areas In First break. Society of Exploration Geophysicists.

[11] Grayver, A. V. (2013). Three-dimensional controlled-source electromagnetic inversion using modern computational concepts. $\mathrm{PhD}$ thesis, Freie Universitä Berlin. 
[12] Hanif, N. H. H. M., Hussain, N., Yahya, N., Daud, H., Yahya, N., and Noh, M. (2011). 1d modeling of controlled-source electromagnetic (csem) data using finite element method for hydrocarbon detection in shallow water. In Proceedings of the International MultiConference of Engineers and Computer Scientists.

[13] Jin, J. (2002). The Finite Element Method in Electromagnetics. Wiley, New York, Second edition.

[14] Key, K. (2012). Marine electromagnetic studies of seafloor resources and tectonics. Surveys in geophysics, 33(1):135-167.

[15] Koldan, J. (2013). Numerical solution of 3-D electromagnetic problems in exploration geophysics and its implementation on massively parallel computers. PhD thesis, Polytechnic University of Catalonia.

[16] Nèdèlec, J. C. (1980). Mixed finite elements in R3. Numerische Mathematik, 35(3), 315-341.

[17] Schoberl, J., Gerstmayr, H., Gaisbauer, R. (2012). NETGENautomatic mesh generator.

[18] Newman, G. A., Commer, M., and Carazzone, J. J. (2010). Imaging CSEM data in the presence of electrical anisotropy. Geophysics, 75(2), F51-F61.

[19] Newman, G. A. (2014). A Review of high-performance computational strategies for modeling and imaging of electromagnetic induction data Surveys in Geophysics, 35(1), 85-100.

[20] OpenMP Architecture Review Board (2015). OpenMP application program interface Version 4.0.

[21] Rognes, M. E., Kirby, R. C., and Logg, A. (2009). Efficient assembly of $\mathrm{H}$ (div) and $\mathrm{H}$ (curl) conforming finite elements. SIAM Journal on Scientific Computing, 31(6), 4130-4151.

[22] Weiss, C. J., Newman, G. A. (2002). Electromagnetic induction in a fully 3-D anisotropic earth. Geophysics, 67(4), 1104-1114.

[23] Weiss, C. J., Constable, S. (2006). Mapping thin resistors and hydrocarbons with marine EM methods, Part II-Modeling and analysis in 3D. Geophysics, 71(6), G321-G332.

[24] Zhdanov, M.S., Lee, S.K., Yoshioka, K. (2006). Integral equation method for 3D modeling of electromagnetic fields in complex structures with inhomogeneous background conductivity. Geophysics 71. 333-345.

[25] Zhdanov, M.S. (2009). Geophysical electromagnetic theory and methods. Elsevier, Amsterdam

[26] Logg A., Mardal, K., Wells, G. (2012). Automated Solution of Differential Equations by the Finite Element Method Springer 